Marquette University

e-Publications@Marquette

$1-1-1997$

\title{
A Descriptive Analysis of the Retail Real Estate Markets at the Metropolitan Level
}

Mark Eppli

Marquette University, mark.eppli@marquette.edu

Steven P. Laposa

Price Waterhouse LLP

Published version. Journal of Real Estate Research, Vol. 14, No. 3 (1997): 321-338. Publisher Link. (C) 1997 American Real Estate Society. Used with permission.

Mark Eppli was affiliated with George Washington University at the time of publication. 


\title{
A Descriptive Analysis of the Retail Real Estate Markets at the Metropolitan Level ${ }^{\dagger}$
}

\author{
Mark J. Eppli* \\ Steven P. Laposa**
}

\begin{abstract}
Gross Leasable Area (GLA) per capita is a commonly used measure to compare the retail market potential across different retail real estate markets. This study uses GLA per capita to assess the supply of the retail space across fifty-eight metropolitan areas in the United States. After a detailed descriptive analysis of the supply of retail space, we estimate GLA per capita for each metropolitan area using a modified version of the stock adjustment model. Initial findings indicate that the retail construction boom of the $1980 \mathrm{~s}$ was not a boom at all and that GLA per capita can be predicted using a multi-factor model.
\end{abstract}

\section{Introduction}

The purpose of this study is to provide a descriptive picture of the retail real estate market and test the viability of the GLA per capita index. Building on several of the inquiries made by Wheaton and Torto (1995), we explore some of the perceived "contradictions" common in retail real estate markets. Using both time-series and cross-sectional data on retail real estate markets, this study tracks fifty-eight retail markets over twenty-seven years. The retail real estate indices are then tested for their ability to predict a good investment market or potential retail location. Our findings are threefold. First, we reveal that the retail construction "boom" of the 1980 s was not a boom at all and that retail construction in the 1970s and 1990s matched that of the 1980s. Second, the research shows that current retail market indices are good indicators of market potential. Lastly, the GLA per capita measure presents a fairly efficient market for retail space across metropolitan areas.

The remainder of the study is as follows. In section two we provide a brief overview of the GLA per capita indice. Section three includes a discussion of the data for each of fifty-eight metropolitan areas using several different definitions of retail space. We then test the predictability of the GLA per capita measure using a variant of the stock adjustment model in the fourth section and close the study with market-level estimates of retail supply per capita.

\section{The Gross Leasable Area per Capita Index}

For an index to be helpful to retail real estate decisionmakers, it must present a meaningful comparison of alternative retail investments/development opportunities across markets. However, a majority of current retail real estate research is focused on the locational and tenant mix factors of retail real estate, where site and tenancy differences

$\dagger 1997$ Retail Estate award, sponsored by the International Council of Shopping Centers.

*Department of Finance, George Washington University, 2023 G Street, N.W., Washington, DC 20052.

**Price Waterhouse LLP, 950 17th Street, Suite 2500, Denver, Colorado 80202. 
make indexing retail real estate difficult if not impossible. ${ }^{1}$ Highlighting the importance of location, some researchers use gravity models to measure the success of a retail location, suggesting that retail sales are a function of center size and travel distance to the center. $^{2}$

Other advanced theoretical models of retail sales allow for the more complex decisionmaking of today's consumer, but these models often require a set of empirical inputs that are either not observable or are not collected. ${ }^{3}$ The use of Geographic Information Systems (GIS) epitomizes the locational importance of a shopping center by modelling the spatial relationships between and among consumers and retailers. ${ }^{4}$ However, GIS modelling has revealed that shopping center developers efficiently locate centers across a metropolitan area, making retail space a commodity when compared across similar center types, which in turn, should make retail real estate imminently indexable.

Gross Leasable Area (GLA) per capita is one method of comparing retail investment potential across markets. This method measures the potential success of a retail location by standardizing retail supply by population, which is described as:

$$
G L A P C_{i}=\frac{G L A_{i}}{P O P_{i}},
$$

where:

$G L A P C_{i}=$ gross leasable area per capita in market area $i$;

$G L A_{i}=$ total retail space in market area $i$; and

$P O P_{i}=$ total population in market area $i$.

The GLA per capita measure has been the industry's darling when discussing the overbuilding of retail space in the mid-1980s, because it standardizes the growth in supply of retail space across metropolitan areas and is easy to use.

\section{A Descriptive Discussion of the Retail Markets}

\section{Retail Real Estate Data, An Overview}

One possible reason for the contradictory evidence on retail real estate may be attributable to retail market data. Retail real estate data problems and limitations are many. For instance, retail properties classified as neighborhood centers in one market are not necessarily classified as neighborhood in another market; retail vacancy and rental rates, if reported, are usually composite statistics that include all retail subproperty types (i.e., anchor and nonanchor tenants); time-series retail data is frequently not consistent across markets and when it is available it is usually for short durations of three to five years, which is not adequate to develop time-series models of supply; and changes in retail supply formats, i.e., power centers, urban malls and discount retail outlets, result in a retail supply that is not easily classified into standard categories.

Industry associations including the Urban Land Institute (ULI) and the International Council for Shopping Centers (ICSC) have promoted standard definitions and classifications of retail properties which has resulted in improved data collection and reporting. Adherence to industry standards will eventually provide the research community with a rich database of retail market data by retail property type. Until such time, 
our understanding of retail property markets will lag the body of knowledge found in office and industrial markets.

\section{FW Dodge Retail Market Data}

A special request to FW Dodge is the primary source of the retail supply data in this study. Annual supply data was produced for fifty-eight markets for 1970 through 1996. Annual retail supply (i.e., construction starts) is divided into two categories-freestanding retail and shopping centers. Each of these categories is further subdivided by shopping center size classifications. Freestanding retail includes non-attached retail construction and freestanding construction in shopping centers (i.e., outpad buildings); the shopping center category includes all retail space that is contiguous. Single restaurant retail sites and food stores such as bakeries, butcher shops and dairy stores are not included in either the freestanding retail or the shopping center data. Other sources of information used in this study include the U.S. Department of Commerce, Woods \& Poole, and the Bureau of Labor Statistics. Exhibit 1 illustrates the type of data provided by each source.

Data provided by FW Dodge has its benefits and limitations. The benefits include consistency of data gathering, time-series data, and metropolitan-level supply statistics. Limitations come in the quality control of the data collected. Classifying FW Dodge's supply statistics by retail classifications (freestanding stores or shopping centers) involves some human judgment. Also, FW Dodge retail supply data does not account for actual space removals or a change from a retail use to another use. To reduce the possibility of data categorization error we combine freestanding and shopping center retail categories into a total retail category when estimating retail supply.

\section{The Stock of Retail Space}

The stock of retail space is a critical input to the GLA per capita measure. To have a meaningful GLA per capita index, an accurate assessment of the supply of retail space is imperative. One of the primary weaknesses of the retail supply data is the accounting for the removals of retail space. Retail space removals as estimated by FW Dodge for the period 1991-96 for fifty-eight MSAs averaged 0.70\% per annum. Discussions with

\section{Exhibit 1}

Data Sources and Variable Descriptions

Source Variable Descriptions

FW Dodge

Annual retail supply (construction starts) for 58 Metropolitan Statistical Areas (MSAs), 1970-96

Retail absorption and vacancy rates for 58 MSAs, 1991-96

US Department of Commerce, Bureau of Economic Analysis, Census Bureau

Woods \& Poole
Annual population, per capita income, retail sales, retail sales excluding automotive expenditures, and number of households, 1969-96

Annual population, number of households, retail sales, and per capita income, 1970-96 
researchers at FW Dodge indicate that they use a nonlinear stock removal function with an assumed ninety-year economic life for retail space. Similarly, Wheaton and Torto (1995) estimate the growth in national retail space without considering any space removals and later use a removal of one percent of the retail stock per annum.

Different from FW Dodge and Wheaton and Torto, a 1973 study by Touche Ross and Company for the International Council of Shopping Centers found that the life of most shopping centers is between twenty-two and twenty-nine years with an average life of twenty-seven years. The study used panel data on 535 shopping centers across the United States. The Touche Ross research found that $69 \%$ of major center alterations for centers fifteen years of age or older were needed to avoid economic obsolescence. Economic obsolescence was measured using factors such as competition, business expansion, changes in merchandising trends, changes in income trends, changes in population density, and changes in driving patterns. Physical decay of the facilities, outdated design, and other unidentified factors accounted for only $31 \%$ of alterations necessary to avoid center obsolescence. While the Touche Ross and Company research is based upon survey data, the "big six" accounting firm presents evidence that the economic obsolescence of a retail center may come much sooner than the physical obsolescence.

While the physical structures that house retail real estate may have a life that approaches ninety or a hundred years, it is likely that the structure will undergo a change in use before the end of the structure's life. A good example of the change in use in retail properties today is culled from the longitudinal data supplied in the ULI's Dollar and Cents of Shopping Centers. Using the ULI's 1969 and 1995 survey results, the percent of shopping center space dedicated to services, which includes financial, offices and personal services, increased $5.6 \%$ (to $16.6 \%$ of space) for neighborhood centers, $4.1 \%$ (to $9.6 \%$ of space) for community centers, $2.0 \%$ (to $5.1 \%$ of space) for regional centers, and $1.7 \%$ (3.9\% of space) for superregional centers. The anecdotal evidence of the ULI survey data supports the findings of the Touche Ross and Company study suggesting that the economic life of a retail center as a retail center may be much shorter than a center's structural life.

Based upon the ULI data and the Touche Ross and Company report the supply of retail space in this analysis only includes retail space that was constructed in the past twenty-seven years, the estimated economic life of a shopping center. While this may have some limiting consequences for the analysis of retail real estate supply, especially for cities with viable downtown retail areas, the stock of space is consistently determined for each market based upon actual (not estimated) retail construction starts data. It should also be noted that the twenty-seven-year average life of a shopping center conveniently matches the time-series of the FW Dodge retail supply data.

\section{GLA per Capita as a Retail Market Benchmark}

The GLA per capita is reported for freestanding retail space, shopping center space and total retail space in Exhibits 2-4. Total freestanding retail space per capita (Exhibit 2) maintains a mean of 11.91 with a range of appoximately 10 to $17 \mathrm{sq}$. $\mathrm{ft}$ per capita with a few notable outliers. All observations of GLA per capita that were less than ten were recorded for established East Coast cities which include Baltimore, Boston, New York City and its subjurisdictions, Philadelphia, Pittsburgh and Washington. Cities that maintained a high GLA per capita, i.e., over eighteen, were all younger, high-growth cities and include Dallas, Denver, Las Vegas, Phoenix, and Salt Lake City. 
Exhibit 2

Gross Leasable Area per Capita for Freestanding Retail Space by Center Size and Metropolitan Area, 1996*

\begin{tabular}{|c|c|c|c|c|c|c|c|c|}
\hline Metro Area & $\begin{array}{c}<10,000 \\
\text { s.f. }\end{array}$ & $\begin{array}{c}10,000 \text { to } \\
25,000 \text { s.f. }\end{array}$ & $\begin{array}{l}25,000 \text { to } \\
100,000 \text { s.f. }\end{array}$ & $\begin{array}{c}100,000 \text { to } \\
200,000 \text { s.f. }\end{array}$ & $\begin{array}{l}200,000 \text { to } \\
400,000 \text { s.f. }\end{array}$ & $\begin{array}{l}400,000 \text { to } \\
800,000 \text { s.f. }\end{array}$ & $\begin{array}{c}>800,000 \\
\text { s.f. }\end{array}$ & $\begin{array}{c}\text { Total } \\
\text { s.f. }\end{array}$ \\
\hline Atlanta & 2.58 & 1.78 & 4.91 & 3.64 & 0.86 & 0.17 & 1.05 & 15.00 \\
\hline Austin & 2.18 & 2.13 & 7.40 & 3.69 & 0.41 & 0.00 & 0.00 & 15.80 \\
\hline Baltimore & 1.48 & 1.25 & 3.13 & 2.66 & 0.79 & 0.00 & 0.00 & 9.31 \\
\hline Birmingham & 2.27 & 1.04 & 4.68 & 2.20 & 1.13 & 0.00 & 0.00 & 11.33 \\
\hline Boston & 1.16 & 0.95 & 2.53 & 1.23 & 0.28 & 0.09 & 0.00 & 6.25 \\
\hline Charlotte & 2.99 & 2.24 & 4.75 & 3.89 & 1.42 & 0.00 & 0.00 & 15.29 \\
\hline Chicago & 1.70 & 1.55 & 3.82 & 3.01 & 0.80 & 0.00 & 0.00 & 10.89 \\
\hline Cincinnati & 2.45 & 1.99 & 4.74 & 3.24 & 0.82 & 0.00 & 0.66 & 13.91 \\
\hline Cleveland & 2.41 & 1.73 & 4.46 & 3.64 & 0.10 & 0.32 & 0.00 & 12.65 \\
\hline Columbus & 2.92 & 2.08 & 5.66 & 3.19 & 0.32 & 0.00 & 0.00 & 14.17 \\
\hline Dallas & 2.75 & 2.18 & 8.02 & 4.25 & 0.77 & 0.19 & 0.34 & 18.50 \\
\hline Denver & 2.10 & 3.28 & 9.31 & 4.01 & 1.12 & 0.60 & 0.00 & 20.42 \\
\hline Detroit & 2.47 & 1.63 & 3.86 & 2.30 & 1.11 & 0.00 & 0.00 & 11.37 \\
\hline Ft. Lauderdale & 3.22 & 2.50 & 6.19 & 3.33 & 0.88 & 0.34 & 0.00 & 16.46 \\
\hline Ft. Worth & 3.50 & 2.51 & 7.60 & 3.93 & 0.14 & 0.00 & 0.00 & 17.68 \\
\hline Greensboro & 3.26 & 2.40 & 5.83 & 3.26 & 1.75 & 0.00 & 0.89 & 17.39 \\
\hline Greenville & 2.92 & 1.81 & 5.88 & 2.66 & 0.25 & 0.00 & 0.00 & 13.51 \\
\hline Hartford & 1.62 & 1.63 & 3.90 & 2.67 & 0.60 & 0.00 & 0.00 & 10.42 \\
\hline Honolulu & 2.14 & 2.21 & 4.25 & 3.32 & 3.43 & 0.00 & 1.01 & 16.36 \\
\hline Houston & 3.13 & 1.75 & 5.78 & 3.59 & 0.90 & 0.30 & 0.00 & 15.45 \\
\hline Indianapolis & 3.38 & 2.35 & 5.58 & 4.33 & 1.31 & 0.00 & 0.00 & 16.96 \\
\hline Jacksonville & 3.66 & 2.57 & 6.10 & 3.39 & 0.49 & 0.00 & 0.00 & 16.20 \\
\hline Kansas City & 2.56 & 2.17 & 5.75 & 3.80 & 0.60 & 0.58 & 0.00 & 15.47 \\
\hline Las Vegas & 4.32 & 2.88 & 8.64 & 2.80 & 0.84 & 0.00 & 0.00 & 19.48 \\
\hline Los Angeles & 2.53 & 1.90 & 3.69 & 2.06 & 0.53 & 0.30 & 0.19 & 11.20 \\
\hline Miami & 2.40 & 1.75 & 3.42 & 2.23 & 0.77 & 0.00 & 0.00 & 10.57 \\
\hline Milwaukee & 2.30 & 2.21 & 4.24 & 1.62 & 0.37 & 0.00 & 0.00 & 10.74 \\
\hline Minneapolis & 1.57 & 1.31 & 4.07 & 3.05 & 0.52 & 0.00 & 0.00 & 10.51 \\
\hline Nashville & 4.00 & 2.40 & 6.14 & 3.47 & 1.26 & 0.00 & 0.00 & 17.27 \\
\hline Nassau-Suffolk & 1.39 & 0.68 & 1.90 & 1.04 & 0.71 & 0.00 & 0.00 & 5.71 \\
\hline New Haven & 1.81 & 1.41 & 2.69 & 2.22 & 0.35 & 0.00 & 0.00 & 8.48 \\
\hline New Orleans & 2.57 & 1.41 & 5.13 & 2.67 & 1.04 & 0.00 & 0.00 & 12.82 \\
\hline New York City & 0.49 & 0.28 & 0.55 & 0.38 & 0.23 & 0.00 & 0.00 & 1.92 \\
\hline Newark & 1.22 & 0.87 & 1.46 & 1.21 & 0.58 & 0.00 & 0.00 & 5.33 \\
\hline Norfolk & 2.30 & 1.76 & 3.88 & 1.79 & 0.57 & 0.00 & 0.00 & 10.30 \\
\hline Oakland & 1.45 & 2.33 & 5.16 & 2.83 & 0.44 & 0.00 & 0.00 & 12.21 \\
\hline Oklahoma City & 2.38 & 1.84 & 5.38 & 3.63 & 0.20 & 0.00 & 0.00 & 13.44 \\
\hline Orange County & 2.95 & 3.17 & 6.63 & 3.29 & 0.33 & 0.31 & 0.00 & 16.68 \\
\hline Orlando & 3.31 & 2.47 & 6.69 & 2.96 & 0.49 & 0.00 & 0.00 & 15.92 \\
\hline Philadelphia & 1.15 & 0.79 & 2.44 & 1.91 & 0.49 & 0.13 & 0.00 & 6.92 \\
\hline Phoenix & 3.55 & 2.88 & 8.74 & 3.01 & 0.44 & 0.00 & 0.00 & 18.62 \\
\hline Pittsburgh & 1.43 & 1.02 & 3.33 & 1.52 & 0.54 & 0.00 & 0.00 & 7.85 \\
\hline Portland & 1.93 & 2.73 & 5.89 & 4.53 & 0.78 & 0.00 & 0.00 & 15.86 \\
\hline Raleigh & 1.91 & 1.54 & 5.34 & 2.58 & 0.32 & 0.00 & 0.00 & 11.69 \\
\hline Richmond & 2.59 & 1.51 & 4.90 & 3.51 & 0.45 & 0.00 & 0.00 & 12.97 \\
\hline Riverside & 2.50 & 2.88 & 7.32 & 2.70 & 0.41 & 0.00 & 0.00 & 15.80 \\
\hline Sacramento & 2.02 & 3.57 & 7.52 & 2.51 & 0.14 & 0.00 & 0.00 & 15.76 \\
\hline St. Louis & 1.17 & 1.34 & 4.26 & 3.32 & 0.38 & 0.00 & 0.00 & 10.47 \\
\hline Salt Lake City & 2.28 & 3.16 & 9.21 & 4.27 & 0.31 & 0.00 & 0.00 & 19.22 \\
\hline San Antonio & 2.30 & 1.84 & 5.81 & 3.09 & 0.40 & 0.00 & 0.00 & 13.45 \\
\hline San Diego & 2.91 & 2.79 & 6.57 & 3.77 & 0.37 & 0.00 & 0.00 & 16.40 \\
\hline San Francisco & 1.41 & 1.69 & 2.99 & 1.93 & 1.78 & 1.23 & 0.00 & 11.02 \\
\hline San Jose & 1.77 & 3.12 & 6.06 & 2.73 & 1.02 & 0.77 & 0.00 & 15.46 \\
\hline Seattle & 1.88 & 2.88 & 5.79 & 3.61 & 0.47 & 0.00 & 0.00 & 14.63 \\
\hline Tampa & 3.30 & 2.30 & 6.03 & 3.09 & 0.64 & 0.00 & 0.00 & 15.56 \\
\hline Tulsa & 2.95 & 2.00 & 7.80 & 2.38 & 0.91 & 0.00 & 0.00 & 16.03 \\
\hline Washington & 1.00 & 0.86 & 3.73 & 2.29 & 0.54 & 0.00 & 0.00 & 8.41 \\
\hline West Palm & 3.21 & 2.40 & 4.89 & 2.69 & 1.46 & 0.00 & 0.00 & 14.66 \\
\hline Average & 2.11 & 1.78 & 4.58 & 2.64 & 0.64 & 0.10 & 0.07 & 11.91 \\
\hline
\end{tabular}

*The supply of space is defined as all construction starts for the period 1970-96. 
Exhibit 3

Gross Leasable Area per Capita for Shopping Center Space by Center Size and Metropolitan Area, 1996*

\begin{tabular}{|c|c|c|c|c|c|c|c|c|}
\hline Metro Area & $\begin{array}{c}<10,000 \\
\text { s.f. }\end{array}$ & $\begin{array}{l}10,000 \text { to } \\
25,000 \text { s.f. }\end{array}$ & $\begin{array}{l}25,000 \text { to } \\
100,000 \text { s.f. }\end{array}$ & $\begin{array}{l}100,000 \text { to } \\
200,000 \text { s.f. }\end{array}$ & $\begin{array}{l}200,000 \text { to } \\
400,000 \text { s.f. }\end{array}$ & $\begin{array}{l}400,000 \text { to } \\
800,000 \text { s.f. }\end{array}$ & $\begin{array}{c}>800,000 \\
\text { s.f. }\end{array}$ & $\begin{array}{l}\text { Total } \\
\text { s.f. }\end{array}$ \\
\hline Atlanta & 0.32 & 1.04 & 7.75 & 4.17 & 2.24 & 1.05 & 0.00 & 16.57 \\
\hline Austin & 0.18 & 0.60 & 5.04 & 2.49 & 1.71 & 0.67 & 1.40 & 12.09 \\
\hline Baltimore & 0.24 & 0.52 & 2.60 & 1.96 & 1.62 & 0.72 & 1.39 & 9.05 \\
\hline Birmingham & 0.21 & 0.60 & 3.75 & 3.59 & 2.50 & 1.65 & 0.00 & 12.30 \\
\hline Boston & 0.10 & 0.37 & 1.18 & 1.77 & 1.10 & 0.76 & 0.38 & 5.66 \\
\hline Charlotte & 0.24 & 0.96 & 6.85 & 2.46 & 2.59 & 2.03 & 2.09 & 17.22 \\
\hline Chicago & 0.31 & 0.87 & 2.54 & 1.82 & 2.05 & 1.09 & 1.03 & 9.71 \\
\hline Cincinnati & 0.39 & 0.87 & 2.63 & 2.23 & 2.50 & 0.57 & 0.63 & 9.82 \\
\hline Cleveland & 0.24 & 0.77 & 2.36 & 1.75 & 1.01 & 0.84 & 0.67 & 7.64 \\
\hline Columbus & 0.45 & 1.23 & 4.43 & 2.83 & 1.03 & 0.76 & 0.85 & 11.58 \\
\hline Dallas & 0.43 & 1.26 & 5.63 & 2.44 & 1.71 & 1.12 & 1.41 & 14.00 \\
\hline Denver & 0.13 & 0.75 & 3.82 & 2.71 & 2.21 & 1.10 & 1.37 & 12.09 \\
\hline Detroit & 0.48 & 1.14 & 3.15 & 1.80 & 0.93 & 0.73 & 0.30 & 8.53 \\
\hline Ft. Lauderdale & 0.45 & 1.88 & 8.24 & 6.62 & 3.76 & 0.87 & 3.11 & 24.93 \\
\hline Ft. Worth & 0.75 & 1.53 & 5.11 & 2.24 & 2.95 & 1.47 & 1.07 & 15.12 \\
\hline Greensboro & 0.31 & 0.61 & 5.58 & 2.40 & 0.72 & 1.51 & 1.65 & 12.78 \\
\hline Greenville & 0.23 & 0.79 & 6.63 & 2.66 & 1.33 & 1.64 & 2.34 & 15.62 \\
\hline Hartford & 0.07 & 0.45 & 2.50 & 1.45 & 1.32 & 0.90 & 0.00 & 6.69 \\
\hline Honolulu & 0.03 & 0.15 & 0.35 & 0.54 & 0.39 & 0.00 & 0.00 & 1.46 \\
\hline Houston & 0.48 & 1.37 & 5.81 & 2.84 & 1.70 & 0.72 & 0.00 & 12.92 \\
\hline Indianapolis & 0.36 & 1.01 & 4.70 & 1.92 & 1.16 & 0.00 & 1.50 & 10.65 \\
\hline Jacksonville & 0.43 & 1.09 & 6.73 & 3.52 & 2.98 & 0.00 & 2.35 & 17.10 \\
\hline Kansas City & 0.34 & 0.72 & 4.31 & 2.51 & 0.89 & 1.28 & 2.16 & 12.21 \\
\hline Las Vegas & 0.42 & 1.71 & 5.97 & 3.70 & 2.82 & 1.85 & 0.00 & 16.47 \\
\hline Los Angeles & 0.47 & 0.86 & 1.88 & 1.10 & 0.81 & 0.72 & 0.31 & 6.15 \\
\hline Miami & 0.58 & 1.49 & 4.91 & 3.49 & 2.14 & 2.64 & 0.59 & 15.84 \\
\hline Milwaukee & 0.38 & 0.89 & 2.27 & 1.56 & 0.72 & 0.41 & 0.00 & 6.23 \\
\hline Minneapolis & 0.21 & 0.79 & 3.17 & 2.02 & 1.68 & 0.63 & 1.51 & 10.01 \\
\hline Nashville & 0.44 & 1.14 & 5.55 & 3.65 & 2.99 & 1.39 & 0.00 & 15.16 \\
\hline Nassau-Suffolk & 0.10 & 0.36 & 1.46 & 1.80 & 0.89 & 1.16 & 0.00 & 5.77 \\
\hline New Haven & 0.21 & 0.67 & 1.82 & 3.53 & 0.79 & 0.40 & 1.52 & 8.94 \\
\hline New Orleans & 0.42 & 1.09 & 3.50 & 2.51 & 0.69 & 0.84 & 0.76 & 9.81 \\
\hline New York City & 0.04 & 0.10 & 0.50 & 0.25 & 0.32 & 0.28 & 0.48 & 1.97 \\
\hline Newark & 0.12 & 0.44 & 1.23 & 1.20 & 0.89 & 1.81 & 0.00 & 5.69 \\
\hline Norfolk & 0.26 & 0.89 & 3.48 & 3.01 & 0.66 & 3.00 & 0.71 & 12.01 \\
\hline Oakland & 0.09 & 0.50 & 2.60 & 1.79 & 0.41 & 0.77 & 0.59 & 6.75 \\
\hline Oklahoma City & 0.37 & 1.49 & 4.73 & 3.47 & 1.47 & 1.70 & 2.71 & 15.94 \\
\hline Orange County & 0.32 & 0.82 & 3.78 & 2.43 & 1.56 & 1.13 & 0.00 & 10.04 \\
\hline Orlando & 0.30 & 1.20 & 8.17 & 5.28 & 1.91 & 1.77 & 4.19 & 22.82 \\
\hline Philadelphia & 0.16 & 0.51 & 2.49 & 2.21 & 1.76 & 0.88 & 0.97 & 8.98 \\
\hline Phoenix & 0.32 & 1.19 & 6.06 & 3.85 & 1.59 & 2.42 & 0.51 & 15.94 \\
\hline Pittsburgh & 0.10 & 0.29 & 2.08 & 1.75 & 2.13 & 1.68 & 0.58 & 8.61 \\
\hline Portland & 0.07 & 0.40 & 2.42 & 2.12 & 1.05 & 0.43 & 0.00 & 6.49 \\
\hline Raleigh & 0.19 & 0.67 & 7.55 & 4.06 & 2.01 & 1.47 & 0.85 & 16.80 \\
\hline Richmond & 0.22 & 0.86 & 5.45 & 2.75 & 2.12 & 0.48 & 0.95 & 12.83 \\
\hline Riverside & 0.18 & 0.82 & 3.77 & 2.18 & 1.61 & 0.70 & 0.68 & 9.94 \\
\hline Sacramento & 0.13 & 1.04 & 3.38 & 2.98 & 0.29 & 0.51 & 0.00 & 8.33 \\
\hline St. Louis & 0.15 & 0.59 & 2.11 & 1.18 & 1.14 & 0.24 & 0.00 & 5.41 \\
\hline Salt Lake City & 0.16 & 0.39 & 2.34 & 1.81 & 0.82 & 0.37 & 2.30 & 8.19 \\
\hline San Antonio & 0.31 & 0.75 & 4.22 & 1.89 & 0.88 & 0.62 & 2.33 & 11.00 \\
\hline San Diego & 0.21 & 0.93 & 3.49 & 1.97 & 0.93 & 0.39 & 0.51 & 8.43 \\
\hline San Francisco & 0.04 & 0.17 & 0.75 & 1.15 & 0.30 & 0.27 & 0.00 & 2.68 \\
\hline San Jose & 0.15 & 0.55 & 1.60 & 1.43 & 0.98 & 0.65 & 0.00 & 5.36 \\
\hline Seattle & 0.10 & 0.55 & 2.64 & 1.58 & 0.62 & 0.57 & 0.73 & 6.79 \\
\hline Tampa & 0.44 & 1.48 & 6.59 & 4.16 & 2.07 & 0.53 & 3.03 & 18.30 \\
\hline Tulsa & 0.34 & 1.24 & 4.73 & 4.38 & 1.25 & 0.83 & 2.01 & 14.78 \\
\hline Washington & 0.19 & 0.65 & 3.66 & 2.63 & 1.94 & 2.15 & 2.21 & 13.43 \\
\hline West Palm & 0.42 & 1.79 & 8.70 & 10.40 & 2.81 & 1.21 & 0.00 & 25.33 \\
\hline Average & 0.27 & 0.79 & 3.40 & 2.26 & 1.40 & 0.96 & 0.84 & 9.92 \\
\hline
\end{tabular}

*The supply of space is defined as all construction starts for the period 1970-96. 


\section{Exhibit 4}

Gross Leasable Area per Capita for All Retail Space by
Center Size and Metropolitan Area, 1996*

\begin{tabular}{|c|c|c|c|c|c|c|c|c|}
\hline Metro Area & $\begin{array}{l}<10,000 \\
\text { s.f. }\end{array}$ & $\begin{array}{l}10,000 \text { to } \\
25,000 \text { s.f. }\end{array}$ & $\begin{array}{l}25,000 \text { to } \\
100,000 \text { s.f. }\end{array}$ & $\begin{array}{c}100,000 \text { to } \\
200,000 \text { s.f. }\end{array}$ & $\begin{array}{l}200,000 \text { to } \\
400,000 \text { s.f. }\end{array}$ & $\begin{array}{l}400,000 \text { to } \\
800,000 \text { s.f. }\end{array}$ & $\begin{array}{c}>800,000 \\
\text { s.f. }\end{array}$ & $\begin{array}{l}\text { Total } \\
\text { s.f. }\end{array}$ \\
\hline Atlanta & 2.90 & 2.82 & 12.66 & 7.82 & 3.10 & 1.22 & 1.05 & 31.57 \\
\hline Austin & 2.36 & 2.72 & 12.44 & 6.19 & 2.11 & 0.67 & 1.40 & 27.89 \\
\hline Baltimore & 1.72 & 1.76 & 5.73 & 4.63 & 2.41 & 0.72 & 1.39 & 18.36 \\
\hline Birmingham & 2.49 & 1.64 & 8.43 & 5.79 & 3.64 & 1.65 & 0.00 & 23.64 \\
\hline Boston & 1.26 & 1.32 & 3.71 & 3.00 & 1.38 & 0.85 & 0.38 & 11.90 \\
\hline Charlotte & 3.23 & 3.20 & 11.60 & 6.34 & 4.02 & 2.03 & 2.09 & 32.51 \\
\hline Chicago & 2.01 & 2.43 & 6.36 & 4.83 & 2.85 & 1.09 & 1.03 & 20.60 \\
\hline Cincinnati & 2.84 & 2.86 & 7.37 & 5.47 & 3.33 & 0.57 & 1.29 & 23.73 \\
\hline Cleveland & 2.65 & 2.49 & 6.82 & 5.39 & 1.10 & 1.16 & 0.67 & 20.28 \\
\hline Columbus & 3.37 & 3.30 & 10.09 & 6.03 & 1.35 & 0.76 & 0.85 & 25.75 \\
\hline Dallas & 3.17 & 3.45 & 13.65 & 6.69 & 2.48 & 1.32 & 1.75 & 32.51 \\
\hline Denver & 2.23 & 4.04 & 13.13 & 6.71 & 3.33 & 1.70 & 1.37 & 32.51 \\
\hline Detroit & 2.95 & 2.76 & 7.01 & 4.10 & 2.04 & 0.73 & 0.30 & 19.89 \\
\hline Ft. Lauderdale & 3.68 & 4.37 & 14.43 & 9.95 & 4.64 & 1.21 & 3.11 & 41.39 \\
\hline Ft. Worth & 4.25 & 4.05 & 12.71 & 6.18 & 3.08 & 1.47 & 1.07 & 32.81 \\
\hline Greensboro & 3.57 & 3.01 & 11.41 & 5.66 & 2.46 & 1.51 & 2.54 & 30.16 \\
\hline Greenville & 3.15 & 2.60 & 12.51 & 5.32 & 1.58 & 1.64 & 2.34 & 29.14 \\
\hline Hartford & 1.70 & 2.08 & 6.40 & 4.12 & 1.92 & 0.90 & 0.00 & 17.12 \\
\hline Honolulu & 2.17 & 2.36 & 4.60 & 3.86 & 3.82 & 0.00 & 1.01 & 17.82 \\
\hline Houston & 3.61 & 3.13 & 11.59 & 6.43 & 2.61 & 1.01 & 0.00 & 28.38 \\
\hline Indianapolis & 3.74 & 3.36 & 10.28 & 6.26 & 2.46 & 0.00 & 1.50 & 27.60 \\
\hline Jacksonville & 4.08 & 3.65 & 12.83 & 6.91 & 3.47 & 0.00 & 2.35 & 33.29 \\
\hline Kansas City & 2.91 & 2.89 & 10.06 & 6.31 & 1.49 & 1.85 & 2.16 & 27.67 \\
\hline Las Vegas & 4.74 & 4.59 & 14.60 & 6.50 & 3.66 & 1.85 & 0.00 & 35.94 \\
\hline Los Angeles & 3.00 & 2.76 & 5.57 & 3.16 & 1.35 & 1.02 & 0.49 & 17.35 \\
\hline Miami & 2.98 & 3.24 & 8.33 & 5.72 & 2.91 & 2.64 & 0.59 & 26.41 \\
\hline Milwaukee & 2.69 & 3.09 & 6.51 & 3.19 & 1.09 & 0.41 & 0.00 & 16.98 \\
\hline Minneapolis & 1.78 & 2.10 & 7.24 & 5.07 & 2.20 & 0.63 & 1.51 & 20.53 \\
\hline Nashville & 4.44 & 3.54 & 11.70 & 7.12 & 4.25 & 1.39 & 0.00 & 32.44 \\
\hline Nassau-Suffolk & 1.49 & 1.03 & 3.36 & 2.83 & 1.61 & 1.16 & 0.00 & 11.48 \\
\hline New Haven & 2.02 & 2.08 & 4.51 & 5.74 & 1.14 & 0.40 & 1.52 & 17.41 \\
\hline New Orleans & 2.99 & 2.50 & 8.63 & 5.18 & 1.73 & 0.84 & 0.76 & 22.63 \\
\hline New York City & 0.53 & 0.38 & 1.04 & 0.63 & 0.55 & 0.28 & 0.48 & 3.89 \\
\hline Newark & 1.34 & 1.30 & 2.69 & 2.41 & 1.46 & 1.81 & 0.00 & 11.01 \\
\hline Norfolk & 2.56 & 2.66 & 7.36 & 4.80 & 1.24 & 3.00 & 0.71 & 22.33 \\
\hline Oakland & 1.54 & 2.83 & 7.77 & 4.62 & 0.84 & 0.77 & 0.59 & 18.96 \\
\hline Oklahoma City & 2.75 & 3.33 & 10.12 & 7.10 & 1.67 & 1.70 & 2.71 & 29.38 \\
\hline Orange County & 3.27 & 4.00 & 10.40 & 5.72 & 1.90 & 1.44 & 0.00 & 26.73 \\
\hline Orlando & 3.60 & 3.67 & 14.86 & 8.24 & 2.41 & 1.77 & 4.19 & 38.74 \\
\hline Philadelphia & 1.31 & 1.31 & 4.93 & 4.12 & 2.25 & 1.01 & 0.97 & 15.90 \\
\hline Phoenix & 3.87 & 4.07 & 14.80 & 6.86 & 2.03 & 2.42 & 0.51 & 34.56 \\
\hline Pittsburgh & 1.54 & 1.31 & 5.42 & 3.27 & 2.66 & 1.68 & 0.58 & 16.46 \\
\hline Portland & 1.99 & 3.13 & 8.31 & 6.65 & 1.83 & 0.43 & 0.00 & 22.34 \\
\hline Raleigh & 2.10 & 2.21 & 12.89 & 6.64 & 2.33 & 1.47 & 0.85 & 28.49 \\
\hline Richmond & 2.81 & 2.37 & 10.35 & 6.26 & 2.57 & 0.48 & 0.95 & 25.79 \\
\hline Riverside & 2.68 & 3.70 & 11.09 & 4.87 & 2.02 & 0.70 & 0.68 & 25.74 \\
\hline Sacramento & 2.15 & 4.61 & 10.89 & 5.49 & 0.44 & 0.51 & 0.00 & 24.09 \\
\hline St. Louis & 1.32 & 1.94 & 6.37 & 4.50 & 1.51 & 0.24 & 0.00 & 15.88 \\
\hline Salt Lake City & 2.43 & 3.55 & 11.55 & 6.08 & 1.13 & 0.37 & 2.30 & 27.41 \\
\hline San Antonio & 2.61 & 2.60 & 10.03 & 4.98 & 1.28 & 0.62 & 2.33 & 24.45 \\
\hline San Diego & 3.12 & 3.71 & 10.05 & 5.74 & 1.30 & 0.39 & 0.51 & 24.82 \\
\hline San Francisco & 1.45 & 1.85 & 3.74 & 3.08 & 2.08 & 1.50 & 0.00 & 13.70 \\
\hline San Jose & 1.92 & 3.66 & 7.65 & 4.16 & 2.00 & 1.42 & 0.00 & 20.81 \\
\hline Seattle & 1.97 & 3.44 & 8.43 & 5.19 & 1.08 & 0.57 & 0.73 & 21.41 \\
\hline Tampa & 3.74 & 3.78 & 12.62 & 7.26 & 2.70 & 0.73 & 3.03 & 33.86 \\
\hline Tulsa & 3.29 & 3.24 & 12.52 & 6.76 & 2.15 & 0.83 & 2.01 & 30.80 \\
\hline Washington & 1.19 & 1.51 & 7.39 & 4.92 & 2.48 & 2.15 & 2.21 & 21.85 \\
\hline West Palm & 3.64 & 4.19 & 13.59 & 13.09 & 4.27 & 1.21 & 0.00 & 39.99 \\
\hline Average & 2.38 & 2.57 & 7.98 & 4.90 & 2.04 & 1.06 & 0.91 & 21.84 \\
\hline
\end{tabular}

*The supply of space is defined as all construction starts for the period 1970-96. 
Results of both the shopping center GLA per capita and total retail (combined freestanding and shopping center) GLA per capita analyses are similar to freestanding GLA per capita. Shopping center and total retail GLA per capita maintained a mean of 9.92 and 21.84, respectively. Most MSAs maintained a total retail GLA per capita of 17 to 26 (see Exhibit 4). The clear outlier is New York City with 3.89 sq. $\mathrm{ft}$ of total retail per capita (recall that this analysis only includes retail space constructed since 1970). Several of the rapidly growing or tourist destination MSAs maintain the highest total retail GLA per capita with Fort Lauderdale at 41.39, West Palm Beach at 39.99, Orlando at 38.74, and Las Vegas at 35.95. As tourist destinations, all of these MSA are able to draw retail dollars from other MSAs, and therefore have a justifiably higher GLA per capita.

\section{The Supply of Retail Space over the Last Twenty-Seven Years}

The overbuilding of the 1980s has been documented in the popular press and in the academic real estate literature which generally states that the lack of discipline among all players in the real estate industry created a glut of office, industrial, hotel, and retail space. In Exhibits 5-8 we catalog the additions to retail supply for the combined fifty-eight MSAs over the past twenty-seven years for freestanding retail, shopping center and total retail space. The increase in retail stock is standardized by the total space added for the period 1970 to 1996. For instance, Exhibit 5 reveals that additions to total square feet of freestanding retail for 1994 was greater than any other year with $5.48 \%$ of the stock added

\section{Exhibit 5}

\section{Supply of Freestanding Retail Space as a Percent of Total Freestanding Construction Starts (1970-96) by Center Size and Year of Completion}

\begin{tabular}{|c|c|c|c|c|c|c|c|c|}
\hline Year & $\begin{array}{c}<10,000 \\
\text { s.f. }\end{array}$ & $\begin{array}{l}10,000 \text { to } \\
25,000 \text { s.f. }\end{array}$ & $\begin{array}{c}25,000 \text { to } \\
100,000 \text { s.f. }\end{array}$ & $\begin{array}{l}100,000 \text { to } \\
200,000 \text { s.f. }\end{array}$ & $\begin{array}{l}200,000 \text { to } \\
400,000 \text { s.f. }\end{array}$ & $\begin{array}{l}400,000 \text { to } \\
800,000 \text { s.f. }\end{array}$ & $\begin{array}{l}>800,000 \\
\text { s.f. }\end{array}$ & $\begin{array}{l}\text { Total } \\
\text { s.f. }\end{array}$ \\
\hline 1970 & 3.57 & 4.14 & 2.86 & 4.78 & 6.94 & 12.40 & 29.24 & 4.06 \\
\hline 1971 & 4.01 & 4.56 & 2.86 & 5.43 & 5.76 & 3.24 & 0.00 & 4.03 \\
\hline 1972 & 4.72 & 5.38 & 3.80 & 5.04 & 5.46 & 0.00 & 10.83 & 4.57 \\
\hline 1973 & 4.92 & 5.90 & 3.79 & 4.37 & 6.75 & 8.95 & 0.00 & 4.62 \\
\hline 1974 & 3.97 & 3.92 & 3.23 & 3.81 & 5.08 & 8.31 & 0.00 & 3.72 \\
\hline 1975 & 3.63 & 3.09 & 2.03 & 3.59 & 4.92 & 9.63 & 0.00 & 3.02 \\
\hline 1976 & 4.52 & 3.84 & 2.53 & 2.58 & 1.96 & 0.00 & 0.00 & 3.02 \\
\hline 1977 & 4.91 & 4.47 & 3.48 & 2.76 & 1.03 & 0.00 & 11.37 & 3.61 \\
\hline 1978 & 5.03 & 4.26 & 3.58 & 3.25 & 3.75 & 4.47 & 14.08 & 3.94 \\
\hline 1979 & 3.67 & 3.47 & 3.70 & 3.65 & 3.62 & 0.00 & 0.00 & 3.59 \\
\hline 1980 & 3.00 & 2.38 & 3.19 & 3.06 & 2.45 & 4.69 & 0.00 & 2.96 \\
\hline 1981 & 2.73 & 2.04 & 2.52 & 2.08 & 0.54 & 0.00 & 0.00 & 2.24 \\
\hline 1982 & 2.44 & 1.84 & 2.32 & 1.33 & 1.87 & 0.00 & 0.00 & 1.99 \\
\hline 1983 & 3.17 & 2.53 & 2.44 & 0.73 & 0.56 & 4.11 & 0.00 & 2.11 \\
\hline 1984 & 3.85 & 3.29 & 3.08 & 1.35 & 1.97 & 11.18 & 0.00 & 2.86 \\
\hline 1985 & 4.62 & 4.45 & 4.18 & 2.54 & 3.64 & 0.00 & 0.00 & 3.84 \\
\hline 1986 & 4.57 & 4.30 & 4.31 & 1.77 & 3.90 & 4.25 & 9.75 & 3.80 \\
\hline 1987 & 3.78 & 3.86 & 4.51 & 2.14 & 3.90 & 0.00 & 0.00 & 3.66 \\
\hline 1988 & 3.77 & 3.67 & 3.91 & 2.20 & 4.28 & 14.20 & 0.00 & 3.56 \\
\hline 1989 & 3.74 & 3.40 & 4.17 & 2.52 & 4.36 & 7.07 & 0.00 & 3.62 \\
\hline 1990 & 3.64 & 3.62 & 4.23 & 2.83 & 3.73 & 0.00 & 15.16 & 3.72 \\
\hline 1991 & 2.85 & 2.87 & 4.17 & 3.19 & 2.57 & 3.61 & 0.00 & 3.41 \\
\hline 1992 & 2.45 & 2.98 & 4.65 & 4.31 & 0.55 & 0.00 & 0.00 & 3.70 \\
\hline 1993 & 2.63 & 3.33 & 4.67 & 6.40 & 4.54 & 0.00 & 0.00 & 4.42 \\
\hline 1994 & 3.04 & 4.01 & 5.65 & 8.62 & 5.01 & 0.00 & 0.00 & 5.48 \\
\hline 1995 & 3.35 & 4.07 & 5.42 & 9.64 & 6.14 & 0.00 & 0.00 & 5.47 \\
\hline 1996 & 3.44 & 4.37 & 4.73 & 6.07 & 4.71 & 3.89 & 0.00 & 4.71 \\
\hline Total & 100.00 & 100.00 & 100.00 & 100.00 & 100.00 & 100.00 & 100.00 & 100.00 \\
\hline
\end{tabular}


to the twenty-seven-year total. During an average year $3.7 \%$ of the total retail space was added to the stock of space. The boom in freestanding construction in the 1990s can be explained by the increase in "big box" discount retailers over the past several years. It should also be noted that in intermittent years space was added in the 400,000-to-800,000 and greater-than- 800,000 sq. $\mathrm{ft}$ categories. We suggest that these observations are attributable to the incorrect coding of shopping center data as freestanding retail as we can cite no examples of freestanding retail facilities greater than $400,000 \mathrm{sq}$. $\mathrm{ft}$.

Exhibit 6 presents the increases to the supply of shopping centers for the period 1970-96, standardized by total shopping center space added. The mid-to-late-1980s represented a period of great expansion for shopping centers smaller than $800,000 \mathrm{sq}$. $\mathrm{ft}$. Super-regional mall construction, as represented by the greater-than-800,000 sq. $\mathrm{ft}$ category, underwent its growth cycle in the 1970s. Overall, shopping center supply additions expanded most rapidly during the 1984-89 period, confirming the anecdotal evidence of the 1980 s construction boom. The combined freestanding and shopping center supply additions are presented in Exhibit 7, which reveals a much more muted development boom in the $1980 \mathrm{~s}$.

In Exhibit 8, we analyze the decade-by-decade additions to supply of retail space for all fifty-eight MSAs standardized by average construction for the period 1970-96. Similar to the National Association of Purchasing Managers (NAPMs) index, any decade with a index number of greater than $100 \%$ indicates that a greater proportion of construction

\section{Exhibit 6}

\section{Supply of Shopping Center Retail Space as a Percent of Total Shopping} Center Construction Starts (1970-96) by Center Size and Year of Completion

\begin{tabular}{|c|c|c|c|c|c|c|c|c|}
\hline Year & $\begin{array}{c}<10,000 \\
\text { s.f. }\end{array}$ & $\begin{array}{c}10,000 \text { to } \\
25,000 \text { s.f. }\end{array}$ & $\begin{array}{l}25,000 \text { to } \\
100,000 \text { s.f. }\end{array}$ & $\begin{array}{l}100,000 \text { to } \\
200,000 \text { s.f. }\end{array}$ & $\begin{array}{l}200,000 \text { to } \\
400,000 \text { s.f. }\end{array}$ & $\begin{array}{l}400,000 \text { to } \\
800,000 \text { s.f. }\end{array}$ & $\begin{array}{l}>800,000 \\
\text { s.f. }\end{array}$ & $\begin{array}{l}\text { Total } \\
\text { s.f. }\end{array}$ \\
\hline 1970 & 1.52 & 1.09 & 1.34 & 2.96 & 3.34 & 4.00 & 1.92 & 2.28 \\
\hline 1971 & 2.04 & 2.08 & 2.21 & 3.51 & 2.71 & 4.83 & 5.30 & 3.08 \\
\hline 1972 & 3.00 & 2.71 & 3.09 & 4.91 & 3.80 & 3.46 & 9.35 & 4.14 \\
\hline 1973 & 3.39 & 2.85 & 4.35 & 4.03 & 5.30 & 3.28 & 8.85 & 4.54 \\
\hline 1974 & 2.03 & 2.45 & 3.15 & 2.77 & 2.68 & 4.44 & 9.39 & 3.56 \\
\hline 1975 & 1.98 & 1.65 & 1.75 & 1.82 & 1.40 & 2.59 & 5.07 & 2.08 \\
\hline 1976 & 3.04 & 2.92 & 2.70 & 1.62 & 1.55 & 1.79 & 0.00 & 2.00 \\
\hline 1977 & 4.04 & 3.78 & 3.50 & 2.76 & 2.39 & 1.96 & 0.89 & 2.84 \\
\hline 1978 & 4.81 & 5.40 & 4.93 & 3.71 & 2.75 & 3.88 & 3.02 & 4.11 \\
\hline 1979 & 3.93 & 4.41 & 5.11 & 4.82 & 4.01 & 6.15 & 6.11 & 4.99 \\
\hline 1980 & 2.98 & 3.10 & 3.78 & 3.39 & 0.97 & 4.73 & 4.86 & 3.40 \\
\hline 1981 & 2.77 & 2.67 & 2.80 & 2.62 & 2.01 & 2.56 & 1.07 & 2.47 \\
\hline 1982 & 3.02 & 2.49 & 2.43 & 1.31 & 2.09 & 2.28 & 0.00 & 1.93 \\
\hline 1983 & 4.88 & 4.75 & 4.58 & 2.91 & 3.11 & 0.98 & 2.61 & 3.50 \\
\hline 1984 & 6.55 & 8.05 & 6.72 & 4.99 & 2.94 & 3.45 & 0.00 & 5.01 \\
\hline 1985 & 9.01 & 8.88 & 7.71 & 7.26 & 7.01 & 4.38 & 2.59 & 6.88 \\
\hline 1986 & 8.55 & 8.28 & 7.40 & 6.72 & 6.01 & 4.81 & 2.80 & 6.51 \\
\hline 1987 & 6.84 & 7.35 & 6.56 & 6.58 & 6.54 & 4.87 & 4.73 & 6.31 \\
\hline 1988 & 6.52 & 6.83 & 5.09 & 5.94 & 5.19 & 4.53 & 3.30 & 5.27 \\
\hline 1989 & 5.64 & 5.79 & 5.35 & 5.59 & 6.68 & 5.86 & 3.73 & 5.55 \\
\hline 1990 & 3.97 & 3.44 & 3.62 & 5.02 & 4.99 & 4.14 & 3.36 & 4.16 \\
\hline 1991 & 1.85 & 1.79 & 2.21 & 2.60 & 3.72 & 2.10 & 1.94 & 2.43 \\
\hline 1992 & 1.26 & 1.35 & 1.64 & 2.09 & 2.59 & 1.89 & 0.00 & 1.73 \\
\hline 1993 & 1.91 & 1.29 & 1.90 & 1.66 & 3.12 & 3.56 & 1.16 & 2.07 \\
\hline 1994 & 1.61 & 1.50 & 2.01 & 2.52 & 3.12 & 2.45 & 3.52 & 2.40 \\
\hline 1995 & 1.40 & 1.48 & 1.92 & 3.49 & 5.28 & 6.06 & 6.90 & 3.52 \\
\hline 1996 & 1.45 & 1.60 & 2.18 & 2.38 & 4.68 & 4.98 & 7.53 & 3.24 \\
\hline Total & 100.00 & 100.00 & 100.00 & 100.00 & 100.00 & 100.00 & 100.00 & 100.00 \\
\hline
\end{tabular}




\section{Exhibit 7}

Supply of Total Retail Space as a Percent of Total Construction Starts (1970-96) by Center Size and Year of Completion

\begin{tabular}{|c|c|c|c|c|c|c|c|c|}
\hline Year & $\begin{array}{l}<10,000 \\
\text { s.f. }\end{array}$ & $\begin{array}{l}10,000 \text { to } \\
25,000 \text { s.f. }\end{array}$ & $\begin{array}{l}25,000 \text { to } \\
100,000 \text { s.f. }\end{array}$ & $\begin{array}{c}100,000 \text { to } \\
200,000 \text { s.f. }\end{array}$ & $\begin{array}{l}200,000 \text { to } \\
400,000 \text { s.f. }\end{array}$ & $\begin{array}{l}400,000 \text { to } \\
800,000 \text { s.f. }\end{array}$ & $\begin{array}{c}>800,000 \\
\text { s.f. }\end{array}$ & $\begin{array}{l}\text { Total } \\
\text { s.f. }\end{array}$ \\
\hline 1970 & 3.34 & 3.20 & 2.21 & 3.94 & 4.46 & 4.82 & 3.99 & 3.25 \\
\hline 1971 & 3.79 & 3.79 & 2.58 & 4.55 & 3.66 & 4.67 & 4.90 & 3.60 \\
\hline 1972 & 4.52 & 4.55 & 3.50 & 4.98 & 4.32 & 3.12 & 9.46 & 4.37 \\
\hline 1973 & 4.75 & 4.96 & 4.03 & 4.21 & 5.75 & 3.83 & 8.17 & 4.58 \\
\hline 1974 & 3.75 & 3.47 & 3.19 & 3.33 & 3.43 & 4.81 & 8.68 & 3.65 \\
\hline 1975 & 3.44 & 2.65 & 1.91 & 2.77 & 2.50 & 3.28 & 4.69 & 2.59 \\
\hline 1976 & 4.36 & 3.55 & 2.60 & 2.14 & 1.68 & 1.61 & 0.00 & 2.56 \\
\hline 1977 & 4.82 & 4.26 & 3.49 & 2.76 & 1.97 & 1.77 & 1.68 & 3.26 \\
\hline 1978 & 5.00 & 4.61 & 4.15 & 3.46 & 3.06 & 3.93 & 3.86 & 4.02 \\
\hline 1979 & 3.70 & 3.76 & 4.30 & 4.19 & 3.89 & 5.55 & 5.64 & 4.22 \\
\hline 1980 & 3.00 & 2.58 & 3.44 & 3.21 & 1.43 & 4.73 & 4.49 & 3.16 \\
\hline 1981 & 2.73 & 2.23 & 2.64 & 2.33 & 1.55 & 2.31 & 0.99 & 2.34 \\
\hline 1982 & 2.51 & 2.04 & 2.37 & 1.32 & 2.02 & 2.05 & 0.00 & 1.96 \\
\hline 1983 & 3.36 & 3.22 & 3.35 & 1.73 & 2.31 & 1.29 & 2.41 & 2.74 \\
\hline 1984 & 4.15 & 4.76 & 4.63 & 3.03 & 2.64 & 4.21 & 2.39 & 3.84 \\
\hline 1985 & 5.11 & 5.82 & 5.68 & 4.72 & 5.96 & 3.95 & 3.33 & 5.22 \\
\hline 1986 & 5.01 & 5.53 & 5.63 & 4.06 & 5.36 & 4.75 & 4.37 & 5.03 \\
\hline 1987 & 4.12 & 4.94 & 5.38 & 4.20 & 5.71 & 4.39 & 3.05 & 4.87 \\
\hline 1988 & 4.08 & 4.65 & 4.42 & 3.93 & 4.91 & 5.47 & 3.45 & 4.34 \\
\hline 1989 & 3.95 & 4.14 & 4.67 & 3.94 & 5.96 & 5.98 & 4.25 & 4.50 \\
\hline 1990 & 3.67 & 3.56 & 3.97 & 3.84 & 4.60 & 3.73 & 1.79 & 3.92 \\
\hline 1991 & 2.74 & 2.54 & 3.33 & 2.92 & 3.36 & 2.24 & 0.73 & 2.97 \\
\hline 1992 & 2.31 & 2.48 & 3.37 & 3.29 & 1.96 & 1.71 & 1.07 & 2.81 \\
\hline 1993 & 2.55 & 2.70 & 3.49 & 4.21 & 3.56 & 3.21 & 3.25 & 3.35 \\
\hline 1994 & 2.88 & 3.23 & 4.10 & 5.80 & 3.71 & 2.21 & 3.25 & 4.08 \\
\hline 1995 & 3.13 & 3.27 & 3.93 & 6.80 & 5.54 & 5.47 & 6.38 & 4.73 \\
\hline 1996 & 3.21 & 3.52 & 3.64 & 4.37 & 4.69 & 4.88 & 6.96 & 4.04 \\
\hline Total & 100.00 & 100.00 & 100.00 & 100.00 & 100.00 & 100.00 & 100.00 & 100.00 \\
\hline
\end{tabular}

\section{Exhibit 8}

Additions to the Supply of Total Retail Space by Decade as a Percent of Total Retail Construction Starts (1970-96)

\begin{tabular}{|c|c|c|c|c|c|c|c|c|}
\hline Year & $\begin{array}{l}<10,000 \\
\text { s.f. }\end{array}$ & $\begin{array}{l}10,000 \text { to } \\
25,000 \text { s.f. }\end{array}$ & $\begin{array}{l}25,000 \text { to } \\
100,000 \text { s.f. }\end{array}$ & $\begin{array}{l}100,000 \text { to } \\
200,000 \text { s.f. }\end{array}$ & $\begin{array}{l}200,000 \text { to } \\
400,000 \text { s.f. }\end{array}$ & $\begin{array}{l}400,000 \text { to } \\
800,000 \text { s.f. }\end{array}$ & $\begin{array}{c}>800,000 \\
\text { s.f. }\end{array}$ & $\begin{array}{l}\text { Total } \\
\text { s.f. }\end{array}$ \\
\hline \multicolumn{9}{|c|}{ Freestanding Retail Space } \\
\hline $\begin{array}{l}1970 \mathrm{~s} \\
1980 \mathrm{~s} \\
1990 \mathrm{~s}\end{array}$ & $\begin{array}{l}1.16 \\
0.96 \\
0.83\end{array}$ & $\begin{array}{l}1.16 \\
0.86 \\
0.97\end{array}$ & $\begin{array}{l}0.86 \\
0.93 \\
1.29\end{array}$ & $\begin{array}{l}1.06 \\
0.53 \\
1.58\end{array}$ & $\begin{array}{l}1.22 \\
0.74 \\
1.05\end{array}$ & $\begin{array}{l}1.27 \\
1.23 \\
0.29\end{array}$ & $\begin{array}{l}1.77 \\
0.26 \\
0.95\end{array}$ & $\begin{array}{l}1.03 \\
0.83 \\
1.20\end{array}$ \\
\hline \multicolumn{9}{|c|}{ Shopping Center Retail Space } \\
\hline $\begin{array}{l}1970 \mathrm{~s} \\
1980 \mathrm{~s} \\
1990 \mathrm{~s}\end{array}$ & $\begin{array}{l}0.80 \\
1.53 \\
0.52\end{array}$ & $\begin{array}{l}0.79 \\
1.57 \\
0.48\end{array}$ & $\begin{array}{l}0.87 \\
1.41 \\
0.60\end{array}$ & $\begin{array}{l}0.89 \\
1.28 \\
0.76\end{array}$ & $\begin{array}{l}0.81 \\
1.15 \\
1.06\end{array}$ & $\begin{array}{l}0.98 \\
1.04 \\
0.97\end{array}$ & $\begin{array}{l}1.35 \\
0.69 \\
0.94\end{array}$ & $\begin{array}{l}0.91 \\
1.26 \\
0.75\end{array}$ \\
\hline \multicolumn{9}{|c|}{ Total Retail Space } \\
\hline $\begin{array}{l}1970 \mathrm{~s} \\
1980 \mathrm{~s} \\
1990 \mathrm{~s}\end{array}$ & $\begin{array}{l}1.12 \\
1.03 \\
0.79\end{array}$ & $\begin{array}{l}1.05 \\
1.08 \\
0.82\end{array}$ & $\begin{array}{l}0.86 \\
1.14 \\
0.99\end{array}$ & $\begin{array}{l}0.98 \\
0.88 \\
1.20\end{array}$ & $\begin{array}{l}0.94 \\
1.02 \\
1.06\end{array}$ & $\begin{array}{l}1.01 \\
1.06 \\
0.90\end{array}$ & $\begin{array}{l}1.38 \\
0.66 \\
0.94\end{array}$ & $\begin{array}{l}0.97 \\
1.03 \\
1.00\end{array}$ \\
\hline
\end{tabular}


occurred in that decade than for other decades. For the total square feet of freestanding retail space constructed category, the 1970 s maintained $103 \%$ of an average year while the 1980s saw little construction of freestanding retail at only $83 \%$ of an average year, and the 1990 s reveal an increase in freestanding retail space constructed to $120 \%$ of the average year. Total shopping center supply additions reveal the converse with $91 \%, 126 \%$ and $75 \%$ of the average for the 1970 s, 1980 s and 1990 s, respectively.

Of particular interest in Exhibit 8 is the decade-by-decade increase in total retail space. The results show that total retail space added during the 1970 s was $97 \%$ of the twentyseven-year average, and during the 1980 s and 1990 s the construction was $103 \%$ and $100 \%$ of the twenty-seven-year average. The total retail space summary statistics reveal that retail construction on a decade-by-decade basis has been flat. ${ }^{5}$ Additionally, if these decade-by-decade numbers were adjusted for the increase in population in the United States, they would certainly show that retail construction has decreased over time on a per capita basis. This decade-by-decade descriptive analysis dispels one retail contradiction-that retail supply has been growing at a more rapid pace in recent yearswhich is not true based on these findings.

\section{Testing the GLA per Capita Index using a Stock Adjustment Model}

\section{The Stock Adjustment Model}

To examine the demand for retail space we adopt a special case of the stock adjustment model that is modified for cross-sectional estimation. There are two reasons for introducing a time-series model that will be tested cross-sectionally. First, stock adjustment models have been the preferred means of modeling additions to the supply of real estate. Other real estate research that has used a version of the stock adjustment model include: Benjamin, Jud and Okoruwa (1993); Benjamin, Jud and Winkler (1996); Eppli and Shilling (1995); Eppli, Shilling and Vandell (forthcoming); Voith and Crone (1988); and Wheaton and Torto (1990). Second, we are attempting to estimate the change in the stock of retail space for the period 1970-96, which follows the theoretical constructs of a stock adjustment model. A stock adjustment model for retail real estate assumes that the desired level of demand for retail space is dependent on retail sales, that is:

$$
K_{i t}^{*}=\frac{S_{i t}}{\Phi_{i}},
$$

or

$$
K_{i t}^{*}=\beta_{i 0}+\beta_{i 1} S_{i t}+\varepsilon_{i t},
$$

where:

$K_{i t}{ }^{*}=$ the desired level retail stock of space in market $i$ at time $t$;

$S_{i t}=$ retail expenditures in market $i$ at time $t$;

$\Phi_{i}=$ equilibrium retail sales per square foot in market $i$; and

$\varepsilon_{i t}=$ random error term. 
$\Phi_{i}$ could also be defined as the number of persons (or fraction of a person) necessary to justify another square foot of retail space. The subscript $i$ refers to each of the fifty-eight metropolitan areas for which we have data. From Pindyck and Rubinfeld (1991) we propose the following stock adjustment model:

$$
K_{i t}-K_{i t-1}=\delta_{i}\left(K_{i t}^{*}-k_{i t-1}\right)
$$

where:

$$
\begin{aligned}
K_{i t} & =\text { the actual stock of retail space in market } i \text { at time } t ; \text { and } \\
\delta_{i} & =\text { the speed of adjustment parameter. }
\end{aligned}
$$

In any given period, the actual value of $K$ may not adjust completely to the desired level of supply. Thus the speed of adjustment factor, $\delta$, is responsible for the partial adjustment and its value is constrained to the range $0<\delta<1$. If the value of $\delta$ approaches 0 , the time it takes for the market to adjust to additions in supply is large. Conversely, if $\delta=1$, the market is able to completely absorb all new space in the period that it is constructed. Substituting equation 4 for $K_{i t}{ }^{*}$ in equation 5 returns:

$$
K_{i t}=\delta_{i} \beta_{i 0}+\delta_{i} \beta_{i 1} S_{i t}+\left(1-\delta_{i}\right) K_{i t-1}+\delta \zeta_{i t}
$$

Data limitations pose direct limitations on our ability to test each metropolitan area for its speed in absorbing new space using equation 6 . The time-series data for each market is twenty-seven periods. Additionally, to test for the speed of adjustment, several lag periods are necessary, further decreasing the number of time-series observations. To address these limitations, we assume that all space is absorbed in the current period by constraining $\delta$ to 1 , and we use a single-period model such that $K_{i t}$ is the amount of additional supply added during the 1970-96 period.

Constraining $\delta$ to 1 is a significant assumption that reduces the time-series stock adjustment model to a cross-sectional model, which is necessary given the data restrictions. ${ }^{6}$ The primary purpose of stock-adjustment models in real estate has been to estimate time lags between when space is delivered and when it is absorbed. However, this research does not focus on the stock operands and instead focuses on the non-stock operands to test the effectiveness of the GLA per capita rule. ${ }^{7}$ Replacing $\delta$ with 1 , equation 6 becomes:

$$
K_{i t}=\beta_{i 0}+\beta_{i 1} S_{i t}+\varsigma_{i l}
$$

In our estimates of the net supply of space using a single-period model, we let $K_{\text {it }}$ represent the change in supply over the past twenty-seven years, which follows the finding of Touche Ross and Company of a twenty-seven-year shopping center economic life.

\section{The Data and Empirical Analyses}

Summary statistics for the data used to test equation 6 are provided in Exhibit 9. For the average metropolitan area more than 50 million sq. $\mathrm{ft}$ of retail space was added between 


\section{Exhibit 9 \\ Summary Statistics}

\begin{tabular}{lrrrr}
\hline Characteristic & Mean & Std Dev. & Min. & Max. \\
\hline $\begin{array}{l}\text { Change in Square Feet of Retail } \\
\text { Space in 1970 to 1996 (in 000) }\end{array}$ & 50,350 & 30,757 & 15,634 & 159,179 \\
$\begin{array}{l}\text { Change in Occupied Square Feet of } \\
\text { Retail Space in 1970 to 1996 (in 000) }\end{array}$ & 46,332 & 28,571 & 14,258 & 150,868 \\
$\begin{array}{l}\text { Change in Retail Sales 1970 to 1996 } \\
\text { (in 000,000) }\end{array}$ & 8,304 & 5,027 & $12,006)$ & 20,144 \\
Total Retail Sales in 1996 & 19,888 & 13,697 & 6,455 & 69,225 \\
Population in 1996 & 2,305 & 1,804 & 747 & 9,138 \\
$\begin{array}{l}\text { Tourism as a Perrcent of Gross } \\
\text { Regional Product in 1996 }\end{array}$ & $4.71 \%$ & $3.03 \%$ & $2.22 \%$ & $23.9 \%$ \\
\hline
\end{tabular}

1970 and 1996. The amount of retail space added varied between 15 and 159 million sq. ft. The change in nominal retail sales between 1970 and 1996 was positive for all metropolitan areas except New York City (not inclusive of its subjurisdictions). The average metropolitan area had total retail sales of $\$ 19.9$ billion and a population of 2.3 million. Tourism as a percent of gross regional product maintained a mean value of $4.71 \%$, with Las Vegas realizing $23.9 \%$ of its gross regional product from tourism in 1996 .

Using the modified stock adjustment model presented in equation 6, we estimate numerous OLS models. The dependent variable is the change in the stock of retail space from 1970-96 across fifty-eight metropolitan areas. $K_{i t}$ is estimated using both the total square feet and the occupied square feet. ${ }^{8}$ Two independent variables are used to estimate the stock of space: the change in retail sales (1970-96) and total retail sales in 1996. Exhibit 10 reveals that the change in retail sales and total retail sales explain a majority of the variance in the stock of retail space. Combining the change in retail sales and total retail sales variables in a single model, approximately $86 \%$ of the variation in supply additions is explained. Exhibit 11 uses the same dependent and independent variables as Exhibit 10, but adjusts the stock of space for depreciation using the Touche Ross and Company findings. ${ }^{9}$ Generally speaking the results show little change using depreciated versus undepreciated stock numbers.

To directly test the GLA per capital model, we standardize equation 6 by population for each metropolitan area which returns the GLA per capita as the dependent variable. Equation 6 is re-estimated and the results are presented in Exhibit $12 .{ }^{10}$ The estimates of GLA per capita show that approximately two-thirds of the variance can be explained by the change in retail sales per capita and total retail sales per capita across both depreciated and undepreciated supply models.

\section{Estimates of GLA per Capita}

Using the results from Exhibit 12 we estimate the desired level of GLA per capita for each MSA. Exhibit 13 presents the results for each market. ${ }^{11}$ Exhibit 13 reveals that the difference between the predicted GLA per capita and actual per capita results is relatively 


\section{Exhibit 10 \\ Explanatory Power of Retail Sales Variables on Total and Occupied Retail Space across 58 Metropolitan Areas, 1996 \\ (t-ratios in parentheses)}

\begin{tabular}{|c|c|c|c|c|}
\hline Dependent Variable & Constant & $\begin{array}{l}\text { Change in Retail Sales, } \\
\qquad 1970-96\end{array}$ & $\begin{array}{c}\text { Total Retail Sales, } \\
1996\end{array}$ & $R$-Squared \\
\hline Total Retail Space & $\begin{array}{l}7,120 \\
(1.71)\end{array}$ & $\begin{array}{r}5.21 \\
(12.12)\end{array}$ & - & $72.4 \%$ \\
\hline Total Retail Space & $\begin{array}{r}13,824 \\
(3.33)\end{array}$ & - & $\begin{array}{r}1.84 \\
(10.64)\end{array}$ & 66.9 \\
\hline Total Retail Space & $\begin{array}{r}1,032 \\
(0.33)\end{array}$ & $\begin{array}{r}3.41 \\
(8.60)\end{array}$ & $\begin{array}{r}1.06 \\
(7.25)\end{array}$ & 85.9 \\
\hline Occupied Retail Space & $\begin{array}{l}6,447 \\
(1.64)\end{array}$ & $\begin{array}{r}4.80 \\
(11.83)\end{array}$ & - & 71.4 \\
\hline Occupied Retail Space & $\begin{array}{r}11,829 \\
(3.17)\end{array}$ & - & $\begin{array}{r}1.73 \\
(11.21)\end{array}$ & 69.2 \\
\hline Occupied Retail Space & $\begin{array}{r}449 \\
(0.16)\end{array}$ & $\begin{array}{r}3.04 \\
(8.45)\end{array}$ & $\begin{array}{r}1.04 \\
(7.89)\end{array}$ & 86.6 \\
\hline
\end{tabular}

aTotal retail space is defined as all construction starts for the period 1970-96. Occupied space is the product of total retail space and the quantity ( 1 minus vacancy rate).

\section{Exhibit 11}

Explanatory Power of Retail Sales Variables on Depreciated Stock of Total and Occupied Retail Space across 58 Metropolitan Areas, 1996

( $t$-ratios in parentheses)

\begin{tabular}{|c|c|c|c|c|}
\hline Dependent Variable & Constant & $\begin{array}{l}\text { Change in Retail Sales, } \\
\qquad 1970-96\end{array}$ & $\begin{array}{c}\text { Total Retail Sales, } \\
1996\end{array}$ & R-Squared \\
\hline Total Retail Space & $\begin{array}{l}3,926 \\
(1,83)\end{array}$ & $\begin{array}{r}2.78 \\
(12.48)\end{array}$ & - & $73.6 \%$ \\
\hline Total Retail Space & $\begin{array}{l}8,264 \\
(3.56)\end{array}$ & - & $\begin{array}{r}0.93 \\
(9.67)\end{array}$ & 62.5 \\
\hline Total Retail Space & $\begin{array}{r}1,076 \\
(0.62)\end{array}$ & $\begin{array}{r}1.92 \\
(8.71)\end{array}$ & $\begin{array}{l}0.494 \\
(6.12)\end{array}$ & 84.3 \\
\hline Occupied Retail Space & $\begin{array}{l}3,562 \\
\langle 1.77\rangle\end{array}$ & $\begin{array}{r}2.54 \\
(12.22)\end{array}$ & - & 72.2 \\
\hline Occupied Retail Space & $\begin{array}{l}7,147 \\
(3.43)\end{array}$ & - & $\begin{array}{r}0.88 \\
(10.17)\end{array}$ & 64.9 \\
\hline Occupied Retail Space & $\begin{array}{r}736 \\
(0.47)\end{array}$ & $\begin{array}{r}1.71 \\
(8.57)\end{array}$ & $\begin{array}{r}0.49 \\
(6.69)\end{array}$ & 85.0 \\
\hline
\end{tabular}

aTotal retail space is defined as all construction starts for the period 1970-96. Occupied space is the product of total retail space and the quantity (1 minus vacancy rate). 


\section{Exhibit 12 \\ Explanatory Power of Models Standardized by Total Population across Total and Occupied Retail Space in 58 Metropolitan Areas, 1996}

( $t$-ratios in parentheses)

\begin{tabular}{|c|c|c|c|c|}
\hline Dependent Variable & Constant & $\begin{array}{c}\text { Change in Retail Sales, } \\
\text { per Capita } \\
1970-96\end{array}$ & $\begin{array}{c}\text { Total Retail Sales, } \\
\text { per Capita } \\
1996\end{array}$ & $R$-Squared \\
\hline \multicolumn{5}{|c|}{ GLA per Capita without Depreciation } \\
\hline Total GLA per Capita & $\begin{array}{r}9.66 \\
(6.02)\end{array}$ & $\begin{array}{r}3.55 \\
(10.06)\end{array}$ & - & $64.4 \%$ \\
\hline Total GLA per Capita & $\begin{array}{l}-8.105 \\
(-1.01)\end{array}$ & - & $\begin{array}{r}3.66 \\
(4.11)\end{array}$ & 23.2 \\
\hline Total GLA per Capita & $\begin{array}{l}20.39 \\
(3.21)\end{array}$ & $\begin{array}{r}4.17 \\
(8.37)\end{array}$ & $\begin{array}{r}-1.50 \\
(-1.75)\end{array}$ & 66.2 \\
\hline Occupied GLA per Capita & $\begin{array}{r}9.23 \\
(6.49)\end{array}$ & $\begin{array}{r}3.17 \\
(10.14)\end{array}$ & - & 64.7 \\
\hline Occupied GLA per Capita & $\begin{array}{r}-7.37 \\
(-1.05)\end{array}$ & - & $\begin{array}{r}3.35 \\
(4.27)\end{array}$ & 24.6 \\
\hline Occupied GLA per Capita & $\begin{array}{r}17.53 \\
(3.10)\end{array}$ & $\begin{array}{r}3.65 \\
(8.22)\end{array}$ & $\begin{array}{r}-1.15 \\
(-1.52) \\
\end{array}$ & 66.1 \\
\hline \multicolumn{5}{|c|}{ GLA per Capita with Depreciation } \\
\hline Total GLA per Capita & $\begin{array}{r}4.27 \\
(4.38)\end{array}$ & $\begin{array}{r}2.13 \\
(9.93)\end{array}$ & - & $63.1 \%$ \\
\hline Total GLA per Capita & $\begin{array}{r}-5.19 \\
(-1.06)\end{array}$ & - & $\begin{array}{r}2.06 \\
(3.77)\end{array}$ & 20.3 \\
\hline Total GLA per Capita & $\begin{array}{r}12.69 \\
(3.35)\end{array}$ & $\begin{array}{r}2.62 \\
(8.81)\end{array}$ & $\begin{array}{r}-1.17 \\
(-2.30)\end{array}$ & 66.9 \\
\hline Occupied GLA per Capita & $\begin{array}{r}4.10 \\
(4.64)\end{array}$ & $\begin{array}{r}1.91 \\
(9.83)\end{array}$ & - & 63.3 \\
\hline Occupied GLA per Capita & $\begin{array}{r}-4.75 \\
(-1.08)\end{array}$ & - & $\begin{array}{r}1.89 \\
(3.86)\end{array}$ & 21.0 \\
\hline Occupied GLA per Capita & $\begin{array}{l}11.05 \\
(3.20)\end{array}$ & $\begin{array}{r}2.31 \\
(8.53)\end{array}$ & $\begin{array}{r}-0.97 \\
(-2.08)\end{array}$ & 66.0 \\
\hline
\end{tabular}

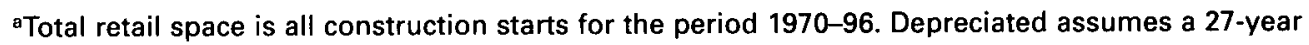
straight-line economic life per Touche Ross and Company (1973).

small indicating that the retail real estate market is relatively efficient. Comparing actual GLA per capita to estimated GLA per capita gives us a benchmark to measure which markets are over/(under) supplied with retail real estate on a relative basis. This analysis can also be completed for each of the market classifications (freestanding retail and shopping centers) and by retail size. For both of the estimated models the over/(under) supply of retail real estate is relatively small with the exceptions of Honolulu and New York, which are both unique retail markets. Finally, if the existing stock of retail real estate can be more accurately measured (especially for the oldest coast cities), the predictive power of the GLA per capita index could be improved. 


\section{Exhibit 13}

\section{Estimates of Total GLA per Capita and Market Supply of Retail Space by Metropolitan Area, 1996}

\begin{tabular}{|c|c|c|c|}
\hline \multirow[b]{2}{*}{ Metro Area } & \multicolumn{3}{|c|}{ Estimates Using Change in Sales per Capita } \\
\hline & $\begin{array}{l}\text { Actual GLA } \\
\text { per Capita }\end{array}$ & $\begin{array}{c}\text { Estimated GLA } \\
\text { per Capita }\end{array}$ & $\begin{array}{l}\text { Market Over/ } \\
\text { (Under) Supply }\end{array}$ \\
\hline Atlanta & 31.57 & 29.70 & 1.87 \\
\hline Austin & 27.89 & 34.08 & -6.19 \\
\hline Baltimore & 18.37 & 19.27 & -0.90 \\
\hline Birmingham & 23.63 & 22.00 & 1.63 \\
\hline Boston & 11.91 & 20.35 & -8.44 \\
\hline Charlotte & 32.52 & 25.70 & 6.82 \\
\hline Chicago & 20.61 & 17.11 & 3.50 \\
\hline Cincinnati & 23.71 & 21.15 & 2.56 \\
\hline Cleveland & 20.30 & 13.78 & 6.52 \\
\hline Columbus & 25.75 & 25.46 & 0.29 \\
\hline Dallas & 32.50 & 29.37 & 3.13 \\
\hline Denver & 32.50 & 27.59 & 4.91 \\
\hline Detroit & 19.89 & 15.94 & 3.95 \\
\hline Ft. Lauderdale & 41.39 & 39.54 & 1.85 \\
\hline Ft. Worth & 32.81 & 28.82 & 3.99 \\
\hline Greensboro & 30.16 & 24.74 & 5.42 \\
\hline Greenville & 29.14 & 23.77 & 5.37 \\
\hline Hartford & 17.12 & 19.68 & -2.56 \\
\hline Honolulu & 17.82 & 30.80 & -12.98 \\
\hline Houston & 28.38 & 27.79 & 0.59 \\
\hline Indianapolis & 27.61 & 22.83 & 4.78 \\
\hline Jacksonville & 33.30 & 26.26 & 7.04 \\
\hline Kansas City & 27.67 & 20.30 & 7.37 \\
\hline Las Vegas & 35.95 & 36.34 & -0.39 \\
\hline Los Angeles & 17.34 & 17.49 & -0.15 \\
\hline Miami & 26.41 & 26.37 & 0.04 \\
\hline Milwaukee & 16.98 & 19.23 & -2.25 \\
\hline Minneapolis & 20.52 & 26.01 & -5.49 \\
\hline Nashville & 32.43 & 29.18 & 3.25 \\
\hline Nassau-Suffolk & 11.48 & 20.47 & -8.99 \\
\hline New Haven & 17.42 & 21.55 & -4.13 \\
\hline New Orleans & 22.62 & 18.88 & 3.74 \\
\hline New York City & 3.89 & 8.83 & -4.94 \\
\hline Newark & 11.01 & 14.79 & -3.78 \\
\hline Norfolk & 22.33 & 23.00 & -0.67 \\
\hline Oakland & 18.95 & 21.92 & -2.97 \\
\hline Oklahoma City & 29.38 & 22.25 & 7.13 \\
\hline Orange County & 26.72 & 28.88 & -2.16 \\
\hline Orlando & 38.73 & 37.44 & 1.29 \\
\hline Philadelphia & 15.90 & 18.14 & -2.24 \\
\hline Phoenix & 34.56 & 31.23 & 3.33 \\
\hline Pittsburgh & 16.47 & 14.88 & 1.59 \\
\hline Portland & 22.35 & 27.42 & -5.07 \\
\hline Raleigh & 28.48 & 28.90 & -0.42 \\
\hline Richmond & 25.79 & 22.36 & 3.43 \\
\hline Riverside & 25.73 & 26.22 & -0.49 \\
\hline Sacramento & 24.09 & 27.37 & -3.28 \\
\hline St. Louis & 15.87 & 18.45 & -2.58 \\
\hline Salt Lake City & 27.41 & 26.10 & 1.31 \\
\hline San Antonio & 24.46 & 25.40 & -0.94 \\
\hline San Diego & 24.83 & 27.17 & -2.34 \\
\hline San Francisco & 13.71 & 21.14 & -7.43 \\
\hline San Jose & 20.82 & 26.41 & -5.59 \\
\hline Seattle & 21.41 & 29.35 & -7.94 \\
\hline Tampa & 33.86 & 31.02 & 2.84 \\
\hline Tulsa & 30.81 & 23.84 & 6.97 \\
\hline Washington & 21.84 & 24.28 & -2.44 \\
\hline West Palm & 39.99 & 38.54 & 1.45 \\
\hline
\end{tabular}




\section{Conclusion}

This study reveals that the retail real estate markets may be more efficient than many pundits suggest. First, the often discussed overbuilding of the retail product in the $1980 \mathrm{~s}$ is a fallacy. The additions to the supply of space in the 1980 s was virtually identical across the decades of the 1970s, 1980s and 1990s. The only difference among decades is the type of retail space constructed (i.e., freestanding or shopping center). Second, the GLA per capita appears to be a viable means of assessing the balance of retail supply and demand across markets. However, direct comparisons across markets need to be adjusted for the supply of retail space built prior to 1970 that is still used as retail space.

\section{Notes}

'See Craig, Ghosh and McLafferty (1984); Eppli and Benjamin (1994); and Ingene (1984); Ingene and Lusch (1980); and Ingene and Yu (1981).

${ }^{2}$ See Eppli and Shilling (1996) and Okoruwa, Nourse and Terza (1994).

${ }^{3}$ The complex decisionmaking by consumers and retailers are partially captured using the Multiplicative Competitive Interaction (MCI) models that are reviewed by Craig et al. (1984).

${ }^{4}$ See Ling and Smersh (1996).

${ }^{5}$ These findings are confirmed by Matzer (1985).

${ }^{6}$ In many ways converting a time-varying model to a cross-sectional model is not satisfying. In an attempt to address this issue, additional analyses were completed using the pooling of cross-section and time-series data (see Pindyck and Rubinfeld, 1991). The results of this estimation procedure, which assigns binary variables to each metropolitan area and each year of analysis were equally unsatisfying. While the predictive power of the model was respectable at $68 \%$, by adding approximately seventy-eight binary variables (the number of binary variables is dependent upon the number of lags in each model) much of the subtlety of the speed of adjustment parameter was lost in the binary variables.

${ }^{7}$ We are thankful to an anonymous reviewer for this helpful clarification.

${ }^{8}$ The generalized definition of the desired stock level incorporates the possibility of a non-zero vacancy rate. We are thankful to an anonymous reviewer's comments for this insight.

${ }^{9}$ Based upon the Touche Ross and Company findings retail centers depreciate on a straight-line basis over a 27 -year economic life.

${ }^{10}$ In addition to the linear OLS estimates shown in Exhibit 12, other tested forms include quadratic, logarithm, exponential, a number of Box-Cox transformations, and the linear form plus binary variables. The results of these additional estimations were similar to the linear form. Additionally, several estimations included an independent variable for tourism as a percent of gross regional product, which generally maintained a negative and insignificant parameter estimate. Our a priori expectations were that this variable would be positive and significant; however, our results may have been skewed by the fact that tourism is $23 \%$ gross regional product for Las Vegas, relative to an average tourism as a percent of gross regional product average of $4.71 \%$. When a binary variable for Las Vegas is included, the tourism as a percent of gross regional product parameter estimate was positive, but remained insignificant.

${ }^{11}$ Using Goldfeld-Quandt (see Pindyck and Rubinfeld, 1991, p. 133) we do not detect the existence of heteroskedasticity. While the empirical tests reveal a homoskedastic relationship, there are several outlier observations including Honolulu and several established East Coast cities. 


\section{References}

Benjamin, J. D., G. D. Jud and A. A. Okoruwa, Forecasting the Stock of Retail Space Using the Koyck Distributed Lag Model, Journal of Property Research, 1993, 10, 185-92.

Benjamin, J. D., G. D. Jud and D. T. Winkler, Forecasting Shopping Center Supply, in J. D. Benjamin, editor, Megatrends in Retail Real Estate, Boston: Kluwer, 1996.

Craig, C. S., A. Ghosh and S. McLafferty, Models of the Retail Location Process: A Review, Journal of Retailing, 1984, 60:1, 5-36.

Eppli, M. J. and J. D. Benjamin, The Evolution of Shopping Center Research: A Review and Analysis, Journal of Real Estate Research, 1994, 9:1, 5-32.

Eppli, M. J. and J. D. Shilling, Speed of Adjustment in Commercial Real Estate Markets, Southern Economic Journal, 1995, 61:4, 1127-45.

- How Critical is a Good Location to a Regional Shopping Center? Journal of Real Estate Research, 1996, 12:3, 459-68.

- and K. D. Vandell, Predictability of Appraisal Based Returns, Journal of Real Estate Finance and Economics, 1996, 16:3.

Ingene, C. A., Structural Determinants of Market Potential, Journal of Retailing, 1984, 60: 1, 37-64. and R. F. Lusch, Market Selection Decisions for Department Stores, Journal of Retailing, 1980, 56:3, 21-40.

Ingene, C. A. and E. S. H. Yu, Determinants of Retail Sales in SMSAs, Regional Science and Urban Economics, 1981, 11, 529-47.

Ling, D. C. and G. T. Smersh, Retail Site Selection Using GIS: An Introduction to Methods, Models, and Data Sources, in J. D. Benjamin, editor, Megatrends in Retail Real Estate, Boston: Kluwer. 1996.

Matzer, J. W., Where Are All Those Older Shopping Centers? in The ICSC Guide to Operating Shopping Centers the Smart Way: Ideas from Industry Experts, New York: International Council of Shopping Centers, 1985.

Okoruwa, A. A., H. O. Nourse and J. V. Terza, Estimating Sales for Retail Centers: An Application of the Poisson Gravity Model, Journal of Real Estate Research, 1994, 9:1, 85-97.

Pindyck, R. S. and D. L. Rubinfeld, Econometric Models and Economic Forecasts, New York: McGraw-Hill, third edition 1991.

Touche Ross and Company, Depreciable Lives of Shopping Centers, an independent study prepared for the International Council of Shopping Centers, New York: ICSC, 1973.

Urban Land Institute, Dollars and Cents of Shopping Centers, Washington, D.C.: ULI, 1969, 1995.

Voith, R. and T. Crone, National Vacancy Rates and the Persistence of Shocks in U.S. Office Markets, AREUEA Journal, 1988, 16:4, 437-48.

Wheaton, W. C. and R. G. Torto, Retail Sales and Retail Real Estate, Real Estate Finance, 1995, 5, 112-39.

, An Investment Model of the Demand for Industrial Real Estate, AREUEA Journal, 1990, $18: 4,530-47$.

The authors would like to thank Luis Mejia of The George Washington University for his assistance with the quantitative analysis portion of this study and Eliza Webb for her editorial comments. We would also like to thank two anonymous reviewers who supplied us with numerous thoughtful comments on the study. 